\title{
Miniature telemetry system for the recording of action and field potentials
}

\author{
Chia-Nan Chien, Fu-Shan Jaw* \\ Institute of Biomedical Engineering, College of Engineering and College of Medicine, National Taiwan University, \\ No.1, Sec. 4, Roosevelt Road, Taipei, Taiwan
}

Received 1 November 2004; received in revised form 21 March 2005; accepted 22 March 2005

\begin{abstract}
A simple miniature telemetry system for neural recording from freely moving rats is described. It weighs only $1 \%$ of the body weight of an adult rat and its recordings are devoid of artifacts due to the animal movement. Together with its long recording time (more than $38 \mathrm{~h}$ ), its isotropic nature, which is essential for working with freely moving animals, offers further advantages. A frequency-modulation receiver with a flat frequency response down to $6 \mathrm{~Hz}$ has been designed for wide-spectrum recording of neural signals, allowing field potential recordings.

A detailed printed-circuit layout and the lack of a trimming requirement will allow the system to be easily duplicated by other neuroscientists who are not familiar with wireless-transmission technologies.
\end{abstract}

(C) 2005 Elsevier B.V. All rights reserved.

Keywords: Action potential; Field potential; Rat; Telemetry

\section{Introduction}

Although a large number of electrophysiological techniques are available to investigate the neural basis of behavior, the physical restraint of the animals is often a major constraint in this type of technically demanding studies. It is highly desirable in some experiments to have the animal free from the encumbrance and restriction of connecting wires and to record from freely moving animals. In this case, the use of telemetry could be attractive and might lead to a new perspective for combined behavioral/electrophysiological approaches.

Biotelemetry, which is the wireless transmission of signals via radio frequency (rf), has fascinated researchers in biomedical science for several decades and is widely applied to convey bioelectric potentials, such as electrocardiograms, electromyograms, and electroencephalograms. Despite the fact that no connecting wires are needed, loading a telemeter built using conventional electronic components onto a conscious rat is difficult due to its dimensions and weight.

Several wireless data-transmission techniques have been developed for recording physiological signals (Skutt et al.,

\footnotetext{
* Corresponding author. Tel.: +8862 23687401; fax: +886233665268.

E-mail address: jaw@ha.mc.ntu.edu.tw (F.-S. Jaw).
}

1967; Fischer et al., 1996; Grohrock et al., 1997; Obeid et al., 2004; Xu et al., 2004). Most of these devices are not suitable for small animals as the components of the transmitter units are relatively large. Therefore, the first step for correlating neural activities to behavior in smaller animals is to construct a miniature telemetric unit. Several such transmitters have been designed previously (Pinkwart and Borchers, 1987; Sundstrom et al., 1997; Winter et al., 1998; Ando et al., 2002; Hawley et al., 2002; Takeuchi and Shimoyama, 2004), and the continuous improvement of electronic components and integrated circuits (ICs) offers the possibility for the easy construction of compact, yet high-quality, systems. Also, the use of modern surface-mounted devices (SMDs) provides the opportunity for neurophysiologists who have little knowledge of electronics to implement a miniature telemeter with ease.

Although many miniature transmission units have been designed for small animals, they are still confined to the laboratory stage. Furthermore, their low-frequency capabilities are poor and not suitable for recording field potentials (FPs). Considering the pass-band requirements of action potentials (APs) and FPs, it was important to develop such a device for our laboratory; therefore, a simple yet useful rf-telemetry system was designed. Components such as small light SMDs and micropower instrumentation amplifiers (IA) were chosen in order to construct a compact lightweight telemeter 
(only $1.2 \%$ of the body weight of a $400 \mathrm{~g}$ rat). The receiver circuit was implemented by utilizing a monolithic IC with minimal peripheral components. The system was tested for wireless transmission of FPs and APs from freely moving rats. Because of its ease of construction, this system could also be useful in other related research fields.

\section{System design}

For recordings from unanaesthtized animals, the neural signal is usually measured by implanted electrodes, and then amplified, modulated and transmitted by a frequency modulation (FM) transmitter. Therefore, an amplifier, an FM transmitter, and a voltage regulator were used to construct the proposed telemeter. The broadcasting signal is detected by the designed receiver, and then demodulated, amplified and filtered as appropriate, and stored onto a recorder.

\subsection{Telemeter}

The telemeter circuit and components list are shown in Fig. 1A. The circuit consists of an IA, an FM transmitter, and a voltage regulator. In order to minimize its size and weight, all the ICs, capacitors and resistors are of the SMD type, except for the high-frequency transistor $(\operatorname{Tr} 1)$ and the

(A)

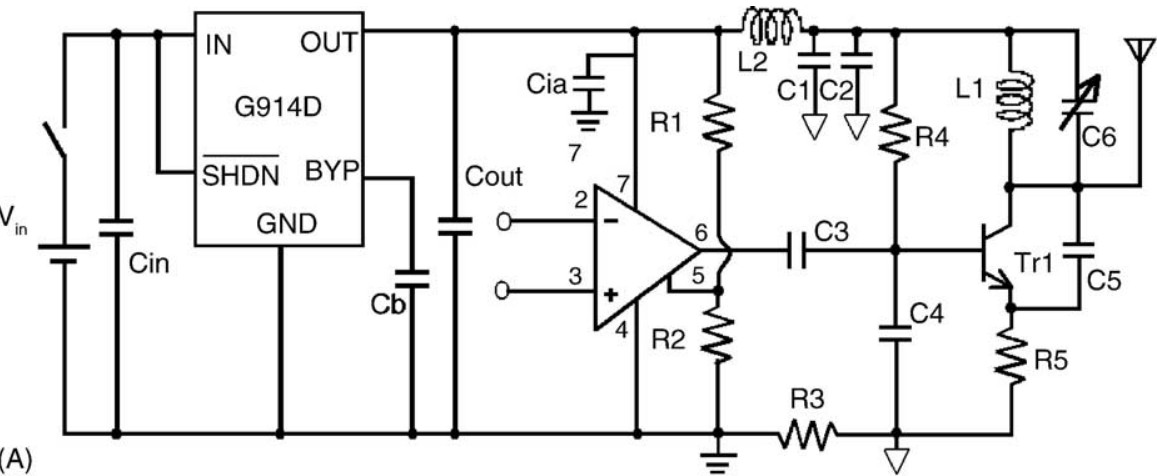

\begin{tabular}{|c|c|c|c|c|c|c|c|}
\hline $\mathrm{R} 1$ & $10 \mathrm{k} \Omega$ & $\mathrm{Rg}$ & $1 \mathrm{k} \Omega$ & $\mathrm{C} 5$ & $15 \mathrm{pF}$ & $\mathrm{Cb}$ & $3.3 \mathrm{nF}$ \\
\hline $\mathrm{R} 2$ & $10 \mathrm{k} \Omega$ & $\mathrm{C} 1$ & $0.1 \mu \mathrm{F}$ & $\mathrm{C} 6$ & $5 \sim 50 \mathrm{pF}$ & $\mathrm{L} 1$ & coil \\
\hline $\mathrm{R} 3$ & $51 \Omega$ & $\mathrm{C} 2$ & $101 \mu \mathrm{F}$ & $\mathrm{Cia}$ & $0.1 \mu \mathrm{F}$ & $\mathrm{L} 2$ & Choke coil \\
\hline $\mathrm{R} 4$ & $47 \mathrm{k} \Omega$ & $\mathrm{C} 3$ & $0.47 \mu \mathrm{F}$ & $\mathrm{Cin}$ & $2.2 \mu \mathrm{F}$ & Tr. & $2 \mathrm{SC} 1359$ \\
\hline $\mathrm{R} 5$ & $680 \Omega$ & $\mathrm{C} 4$ & $1 \mathrm{nF}$ & Cout & $2.2 \mu \mathrm{F}$ & Amp. & AD627 \\
\hline
\end{tabular}
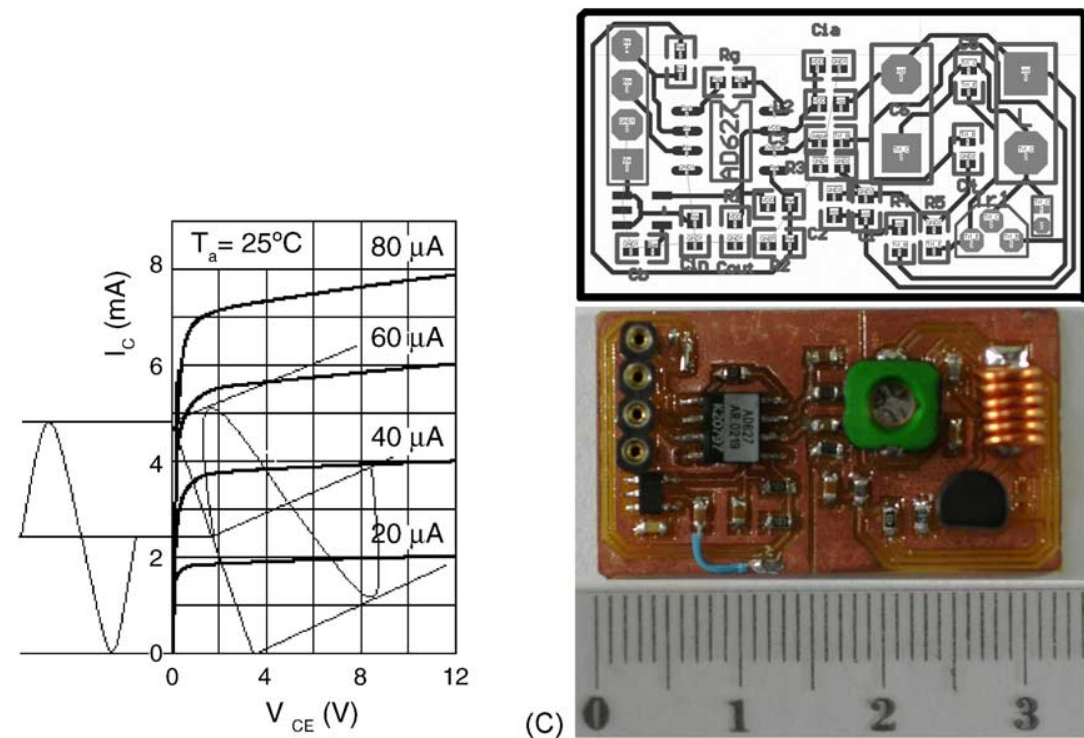

(B)

(C)

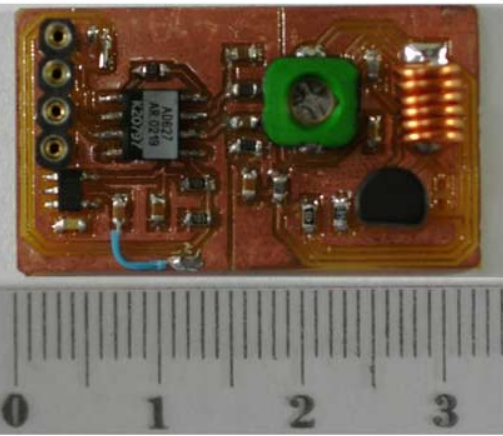

Fig. 1. Circuit diagram of the telemeter. (A) Detailed circuit and components list. (B) Use of the load-line method to set the transistor in the class-A mode of operation; i.e., biased at $2.4 \mathrm{~mA}$. (C) Components and circuit layout of the telemeter $(32.7 \mathrm{~mm} \times 18.8 \mathrm{~mm})$. 
tuning capacitor (C6). To reinforce its mechanical strength, a printed-circuit technique (Fig. 1C) was used to implement the circuit, thus, also facilitating the duplication of the telemeter.

The IA (AD627; Analog Devices) was used before the transmitter to provide adequate gain. The AD627 is an integrated micropower IA that delivers a rail-to-rail output swing on a single supply, providing the user with excellent ac and dc specifications while operating at $85 \mu \mathrm{A}$ (maximum); the gain can be programmed by the $\mathrm{Rg}$ resistor.

Following the IA is the transistor, which serves as an oscillator, modulator, amplifier, and transmitter. The transistor is biased in class-A and is determined by the load-line method (Fig. 1B), as this type of operation is typically used in applications requiring high linearity, high gain, and broadband. The signal is frequency modulated by the modulator circuit (LC-oscillator circuit; based on the Colpitts principle). The carrier frequency can be tuned from 88 to $108 \mathrm{MHz}$, as determined by an air-core coil L1, and the C5 and C6 capacitors. The trimming capacitor C6 allows the carrier to be aligned to a wavelength well distinct from those of nearby radio stations to minimize their interference.

The telemeter circuit is powered by a light-weight commercial hybrid-lithium battery (AHB401430; $100 \mathrm{mAh}$, 3.75 V, $2.2 \mathrm{~g}$; SYNergy ScienTech Corp.). In our design, the battery can be easily removed from the connector for recharging; hence, we can continue the recording with minimal intervention by replacing an exhausted battery with a fully charged one. A low-noise low-dropout linear regulator (G914D; Global Mixed-Mode Technology) was used to stabilize the supply voltage at $3.3 \mathrm{~V}$ to compensate for the voltage drift of the battery. To reduce interference, the decoupling circuit was implanted (L2, C1 and C2 in Fig. 1A) and the grounding of the oscillator was separated from the other parts.

\subsection{Receiver}

The rf signal from the transmitter can be picked up by many methods. A commercial FM tuner covering the passband from 88 to $108 \mathrm{MHz}$ can be used to receive the transmitted signal. However, the signal obtained in this way suffers from a poor low-frequency response. Frequency components lower than $100 \mathrm{~Hz}$ are highly attenuated by commercial FM tuners because they are designed for audio purposes but not for FP recording. To overcome this difficulty, we designed a receiver that could cover the low-frequency band of FPs. An FM receiver IC (TDA7010T; Philips Semiconductors) was used to implement such a receiver (Van and Hufschmidt, 1991). This IC is a monolithic chip that requires minimal peripheral components. The complete circuit of the receiver is shown in Fig. 2. The values of the tuning capacitors, $\mathrm{Cp}$ and

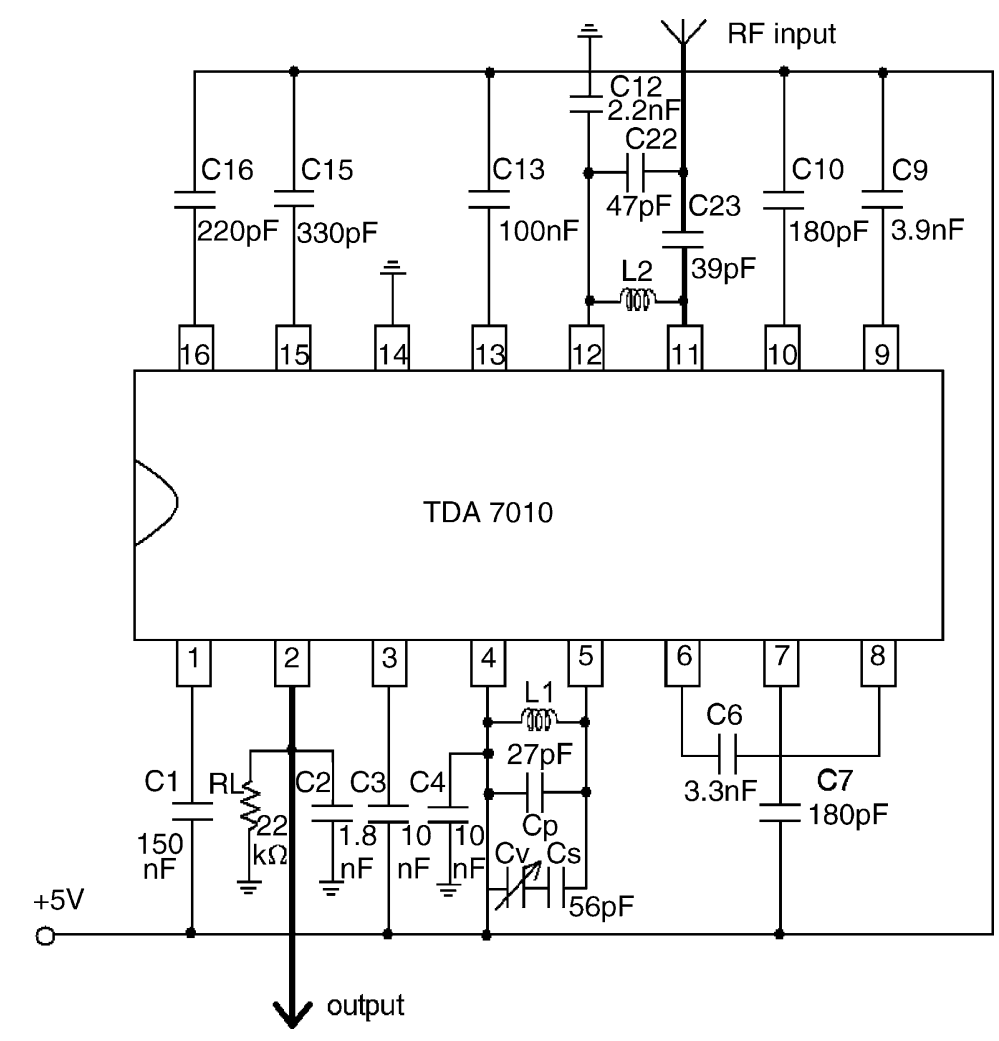

Fig. 2. Complete circuit diagram of the receiver. The carrier frequency of the receiver is adjusted by turning the variable capacitor, Cv. As this circuit is modified from the datasheet of the Philips semiconductor, permission for its use should be requested from the copyright owner. To enable the user to customize the bandwidth, the equations that govern the adjustment of the corner frequencies are given: $f_{0}=1 / 2 \pi R_{1} \sqrt{C_{6} C_{7}}$ where $R_{1}$ is internal $2.2 \mathrm{k} \Omega$ resistor; $f_{\mathrm{HP}}=1 / 2 \pi R_{2} C_{9}$ where $R_{2}$ is internal $4.7 \mathrm{k} \Omega$ resistor and $f_{\mathrm{LP}}=1 / 2 \pi R_{3} C_{10}$ where $R_{3}$ is internal $4.7 \mathrm{k} \Omega$ resistor. 
$\mathrm{Cs}$, depend on the required tuning range and the value of the tuning capacitor $(\mathrm{Cv})$. The values of $\mathrm{C} 22, \mathrm{C} 23, \mathrm{~L} 1$ and L2 given are for an $\mathrm{rf}$ band-pass filter with $Q=4$ for the domestic FM broadcast band (88-108 MHz).

\section{Results}

\subsection{Physical specifications of the telemetry system}

The telemeter occupies a printed-circuit board area of only 32.7 by $18.8 \mathrm{~mm}$ and weighs just $4.84 \mathrm{~g}$. The total supply current of the telemeter is about $2.57 \mathrm{~mA}$ at $3.3 \mathrm{~V}$. The background noise of the amplifier is about $6.8 \mu \mathrm{V} / \sqrt{ } \mathrm{Hz}$ (referto-input, RTI) and the input range of the telemeter is from $25 \mu \mathrm{V}$ to $1 \mathrm{mV}$. The input dc offset range depends on the gain of the amplifier and the supply voltages. At present, the gain of the amplifier is programmed at 200; thus, the input dc offset range is equal to $9 \mathrm{mV}$. The common mode rejection ratio is determined by the gain of the amplifier; theoretically, this should be more than $100 \mathrm{~dB}$. However, the practical performance measured is only $50 \mathrm{~dB}$.

To test the distance of transmission, a $14 \mathrm{~Hz}$ sine wave was used as the signal source. The radiated signal covers isotropically a range of up to $2 \mathrm{~m}$ in radius. Fig. 3 shows the
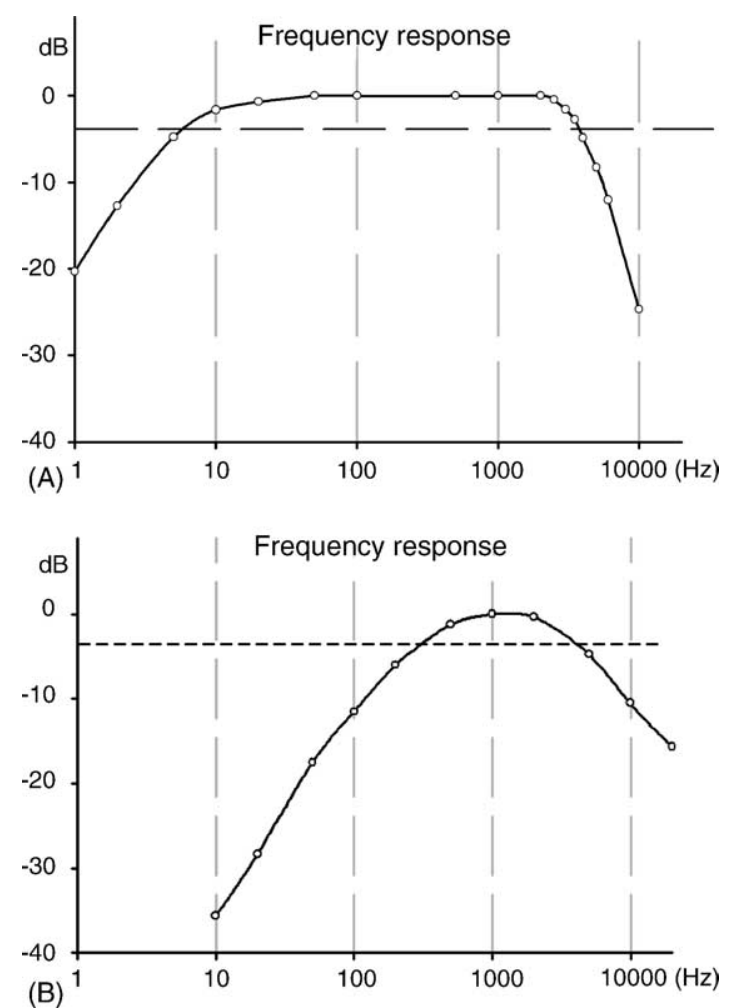

Fig. 3. Responses of the transmission systems. (A) Frequency response of the telemeter-receiver system. The pass-band is from $6 \mathrm{~Hz}$ to $3.8 \mathrm{kHz}$ $(-3 \mathrm{~dB})$, which covers the spectra of most neural signals. (B) The effect of using a commercial FM radio to replace the designed receiver. Notice that the low-frequency corner frequency is at $300 \mathrm{~Hz}$. frequency response of the receiver versus that of a commercial radio. The carriers of the telemeter and receiver were adjusted to the same frequency. During tuning, the received signal can be clearly identified because high-frequency signals are filtered out by the telemetry system with a corner frequency of $3.8 \mathrm{kHz}$. Fig. 3A shows the frequency response of the complete telemetry system; that is, the telemeter plus the receiver. Fig. 3B shows the frequency response using a commercial FM radio receiver instead of our receiver. It is clear that the difference between our receiver and the commercial one at low frequency is relatively large. The FPs are completely filtered out by the commercial receiver (Jaw, 2001).

\subsection{Wireless transmission of neural signals}

After surgical operations under full anesthesia, a Wistar rat was implanted with a microelectrode in the primary somatosensory cortex (Tsai, 2003; Tsai and Yen, 2003) based on the stereotaxic coordinate from Paxinos and Watson (1998). The telemeter circuit was held on the shoulder of the animal with a home-made jacket (Xu et al., 2004), following a standard procedure for animal handling (Shaw et al., 1999).

A preliminary application of this system to neural signals recordings is shown in Fig. 4. As the neuronal APs contain

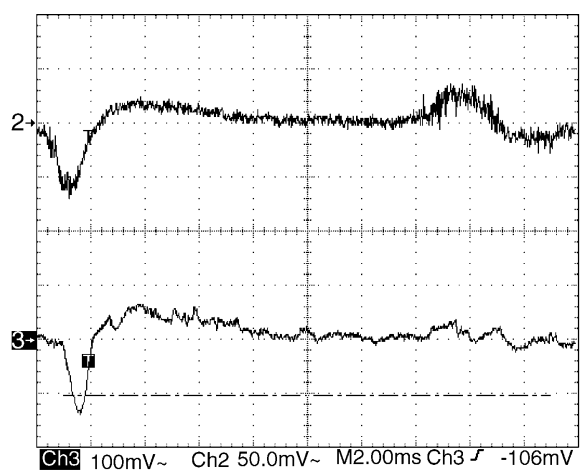

(A)

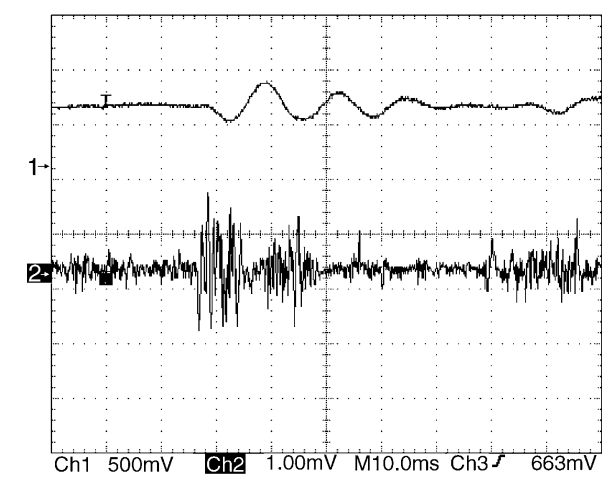

(B)

Fig. 4. Transmission of neural signals. (A) An AP is displayed at high resolution (timebase: $2 \mathrm{~ms}$ ). The upper trace is the waveform before transmission, while the lower is the received waveform. (B) A slower sweep (timebase: $10 \mathrm{~ms}$ ) of the evoked potentials (upper trace) with the extracellular multi-unit response shown below. Different filtering was used in these traces. 
high-frequency content up to about $9 \mathrm{kHz}$ (Jaw, 2001), the pass-band of the system should be sufficiently wide. However, traditional multi-unit recordings utilize the pass-band from $300 \mathrm{~Hz}$ to $3 \mathrm{kHz}$ because the high-frequency components of APs are extremely small. Considering the finite roll-off rate of the filter, the error introduced is less than the background fluctuation. Hence, the $3.8-\mathrm{kHz}$ cut-off frequency was selected as a compromise between waveform fidelity and noise. Although there is some amount of distortion of the waveform after transmission (Fig. 4A), this degree of error might be acceptable for extracellular recordings. The evoked neural signals were transmitted at a distance of $1 \mathrm{~m}$. In Fig. 4B, the upper trace represents FPs whereas the lower trace represents multi-unit APs. These were individually filtered with two filters: one with a pass-band from 0.1 to $100 \mathrm{~Hz}$ for recording FPs, and the other from $300 \mathrm{~Hz}$ to $3 \mathrm{kHz}$ for recording APs (Jaw, 2001).

After several trials, the differences between the cable- and wireless-transmitted signals were not too large. It should be noted that the FP signal was not detected if a commercial radio receiver was used instead (data not shown). Hence, our system could be valuable not only for high-frequency APs but also for low-frequency FPs.

\section{Discussion}

Telemetry is a valuable technique that allows the recording of neural signals in conscious, freely moving animals without the use of any connecting wires. Thus, it is particularly useful in studies aimed at correlating neuronal firing with sensitive behavioral paradigms. The telemetry system that we have developed is of low cost and of easy construction, and might, therefore, be useful to other investigators who lack extensive technological facilities. The physical dimensions of the telemeter are suitable for an adult rat, although further reductions in size might be possible. Moreover, being only about $1 \%$ of the body weight of an adult rat, the telemeter caused no obvious 'inconvenience' during the animal movements. The capacity of the battery can steadily supply current to the telemeter for more than $38 \mathrm{~h}$, a duration sufficiently long for the majority of this type of experiments. A six-turn air coil was wound as an antenna. Although it is not tuned to the optimal condition, its isotropic property makes it especially attractive for a freely moving rat. Furthermore, prolonged tuning utilizing an expensive spectrum or network analyzer can be avoided. This is a great advantage, particularly for neuroscientists who are not familiar with the techniques and principles of telecommunication.

For simplicity, a commercial receiver can be used to record APs. It is, however, recommended that the designed receiver should be used if a wide-spectrum recording is necessary, because the frequency components of FPs are lower than $40 \mathrm{~Hz}$ (Jaw, 2001) and could not be recorded using a commercial FM radio. For customer applications and quick reference, the equations governing the bandwidth adjustment are given in the legend of Fig. 2.

Although the literature includes several reports of singleor dual-channel analog neural-telemetry systems, multichannel telemetry systems should be implemented digitally. A micro-controller-based time-division multichannel telemetry system is currently under development in our laboratory (Liao et al., 2004). The aim of this work is to develop a system, from an MEMS-based neuroprobe (Chen et al., 2004) to a real-time analysis system, for the large-scale recording of neuronal activities.

\section{Acknowledgements}

This work was supported by grant 92-EC-17-A-05S10017 from the Ministry of Economic Affairs, Taiwan, ROC. We would like to thank the anonymous reviewers for their very constructive comments.

\section{References}

Ando N, Shimoyama I, Kanzaki R. A dual-channel FM transmitter for acquisition of flight muscle activities from the freely flying hawkmoth, Agrius convolvuli. J Neurosci Methods 2002;115:181-7.

Chen Y-Y, Kuo T-S, Jaw F-S. A laser micromachined probe for recording multiple field potentials in the thalamus. J Neurosci Methods 2004;139:99-109.

Fischer H, Kautz H, Kutsch W. A radiotelemetric 2-channel unit for transmission of muscle potentials during free flight of the desert locust, Schistocerca gregaria. J Neurosci Methods 1996;64:39_ 45.

Grohrock P, Hausler U, Jurgens U. Dual-channel telemetry system for recording vocalization-correlated neuronal activity in freely moving squirrel monkeys. J Neurosci Methods 1997;76:7-13.

Hawley ES, Hargreaves EL, Kubie JL, Rivard B, Muller RU. Telemetry system for reliable recording of action potentials from freely moving rats. Hippocampus 2002;12:505-13.

Jaw F-S. Optimal sampling of electrophysiological signals. Neurosci Res Commun 2001;28:75-84.

Liao F-R, Chen C-A, Lu S-S, Chiu N-F, Lin C-W, Li J-Y, et al. An implantable integrated SiGe FM transmitter for HRV biotelemetry. 2004 IEEE International Workshop on Biomedical Circuits and Systems:Singapore;2004.

Obeid I, Nicolelis MAL, Wolf PD. A multichannel telemetry system for single unit neural recordings. J Neurosci Methods 2004;133: 33-8.

Pinkwart C, Borchers HW. Miniature three-function transmitting system for single neuron recording, wireless brain stimulation and marking. J Neurosci Methods 1987;20:341-52.

Shaw F-Z, Chen R-F, Tsao H-W, Yen C-T. A multichannel system for recording and analysis of cortical field potentials in freely moving rats. J Neurosci Methods 1999;88:33-43.

Skutt HR, Beschle RG, Moulton DG, Koella WP. New subminiature amplifier-transmitters for telemetering biopotentials. Electroenceph Clin Neurophysiol 1967;22:275-7.

Sundstrom LE, Sundstrom KE, Mellanby JH. A new protocol for the transmission of physiological signals by digital telemetry. J Neurosci Methods 1997;77:55-60.

Paxinos G, Watson C. The rat brain: in stereotaxic coordinates. fourth ed. Academic Press; 1998. 
Takeuchi S, Shimoyama I. A radio-telemetry system with a shape memory alloy microelectrode for neural recording of freely moving insects. IEEE Trans Biomed Eng 2004;51:133-7.

Tsai M-L, Yen C-T. A simple method for fabricating horizontal and vertical microwire arrays. J Neurosci Methods 2003;131:107-10.

Tsai Y-C Measurement systems for electrophysiology signals - from basic biomedical recordings to clinical applications. M.S. Thesis, Institute of Biomedical Engineering, National Taiwan University:Taiwan; 2003.
Van WHA, Hufschmidt M. A complete FM radio on a chip. AN192: Philips Semiconductors; 1991.

Winter KF, Hartmann R, Klinke R. A stimulator with wireless power and signal transmission for implantation in animal experiments and other applications. J Neurosci Methods 1998;79:79-85.

Xu S, Talwar SK, Hawley ES, Li L, Chapin JK. A multi-channel telemetry system for brain microstimulation in freely roaming animals. J Neurosci Methods 2004;133:57-63. 